\title{
Black Hole Discharge: very-high-energy gamma rays from black hole-neutron star mergers
}

\author{
Zhen $\operatorname{Pan}^{1, *}$ and Huan Yang ${ }^{1,2, \dagger}$ \\ ${ }^{1}$ Perimeter Institute for Theoretical Physics, ON, N2L2Y5, Canada \\ ${ }^{2}$ University of Guelph, Guelph, Ontario N2L 3G1, Canada
}

(Dated: August 15, 2019)

\begin{abstract}
With mass ratio larger than $\sim 5$ (which depends on the black hole spin and the star radius), star disruption is not expected for a black hole merging with a neutron star during the final plunge phase. In the late inspiral stage, the black hole is likely charged as it cuts through the magnetic field carried by the neutron star, leaving a temporarily charged black hole after merger. The unstable charged state of the remnant black hole rapidly neutralizes by interacting with the surrounding plasma and photons, which we investigate in first principle by numerically solving a coupled set of Boltzmann equations of $1+1$ form for non-spinning BH background. The resulting basic picture is as follows. Electrons and positrons are accelerated in the BH electric field, which then lose energy to surrounding soft photons via Compton scattering; more electrons and positrons will be created from pair production as the hard photons colliding with soft photons, or through the Schwinger process in strong electromagnetic fields. The cascade stops when the charged black hole accretes enough opposite charges and becomes neutralized. We find that $\sim 10 \%$ (which depends on the soft photon energy and number density) of the total electric energy is carried away to infinity in a time interval $\sim 1 \mathrm{~ms}$ by very-high-energy ( $>50 \mathrm{GeV}$, the low energy detection threshold of the MAGIC telescope) gamma rays whose spectrum is approximately a power law with spectral index $\sim-2.3$. We expect the discharge picture to be true for spinning charged BHs as well.
\end{abstract}

\section{INTRODUCTION}

Short gamma-ray burst (sGRB) has long been suggested to be associated with double neutron star (NS) mergers - a conjecture lately supported by the multimessenger detection of gravitational waves $(\mathrm{GWs})$ and various electromagnetic (EM) counterparts in GW170817 $[1,2]$. Another possible origin of sGRBs is merging black hole $(\mathrm{BH})$ and NS binary. If the $\mathrm{BH}$ disrupts the NS during the late inspiral phase, the remnant matter may form a temporary accretion disk around the final $\mathrm{BH}$ and power an energetic jet along with gamma ray emission [3]. This scenario may be tested with imminent detection of $\mathrm{BH} / \mathrm{NS}$ merger events in the O3 run of LIGO/Virgo collaboration, together with EM observations. Future third-generation GW detectors may be able to probe the NS disruption $[4,5]$ and help distinguish possible lowmass $\mathrm{BH} / \mathrm{NS}$ binaries from binary $\mathrm{NSs}[6-8]$. On the other hand, if the $\mathrm{BH} / \mathrm{NS}$ binary mass ratio is beyond certain threshold $\sim 5$ which depends on the $\mathrm{BH}$ spin and the NS radius ([9-12], dubbed as type III merger therein and hereafter), no star disruption is expected. The $\mathrm{BH}$ basically swallows the NS, and the post-merger waveform is dominated by $\mathrm{BH}$ ringdowns [13, 14]. The associated electromagnetic emission during the merger mainly originates from the surrounding plasma within the system, which has a much smaller energy reservoir than a typical sGRB. A few mechanisms that might lighten up these systems have been proposed, e.g., DC circuit

\footnotetext{
* zpan@perimeterinstitute.ca

† hyang@perimeterinstitute.ca
}

[15, 16], $\mathrm{BH}$ pulsar [17], $\mathrm{BH}$ electric generator [18] and electric/magnetic dipole radiation [19, 20]. Previous discussions focused on estimating the energy budget rather than description about the plasma dynamics and emission properties, so that the spectroscopic prediction of EM counterparts has not been made.

In this paper, we report a first-principle study of EM signals from the aftermath of type III BH/NS mergers. Considering a typical NS mass $\sim 1.4 M_{\odot}$, the corresponding $\mathrm{BH}$ mass threshold is already near the lower end of the observed BH mass in X-ray binaries [22-25]. To simplify the set-up, we neglect the BH spin and assume the final $\mathrm{BH}$ to be Schwarzschild. The amount of electric charge carried by the $\mathrm{BH}$ can be roughly estimated as (also see [17]) $Q \approx E M^{2} \approx B_{\mathrm{NS}} r_{\mathrm{NS}}^{2}$, where $M$ is $\mathrm{BH}$ mass and $E \sim\left(v_{\text {rel }} / c\right) B_{\mathrm{NS}} \approx B_{\mathrm{NS}}$ is the magnitude of the induced electric field. ${ }^{1}$ To get an intuition about the $\mathrm{BH}$ charge $Q$, we define a dimensionless quantity

$$
\Gamma_{Q} \equiv \frac{Q e}{M m_{e}},
$$

which is the ratio of electric force and gravitational force on an electron outside the charge $\mathrm{BH}$. For $M=10 M_{\odot}$, $B_{\mathrm{NS}}=10^{12} \mathrm{G}$ and $r_{\mathrm{NS}}=10 \mathrm{~km}$, we have $\Gamma_{Q, 12} \approx 4 \times 10^{14}$ and total electric energy $Q_{, 12}^{2} / 4 M \approx 10^{42} \mathrm{ergs}$, where the lower index ${ }_{12}$ is the power index of $B_{\mathrm{NS}}[\mathrm{G}]$. Electrons and positrons are accelerated in the $\mathrm{BH}$ electric field, which consequently produce hard photons via inverse

\footnotetext{
${ }^{1}$ As shown in [20], the NS also carries net charge $\sim \Omega_{\mathrm{NS}} B_{\mathrm{NS}} r_{\mathrm{NS}}^{3}$ which is smaller than the $\mathrm{BH}$ charge by a factor $\sim \Omega_{\mathrm{NS}} r_{\mathrm{NS}}$, where $\Omega_{\mathrm{NS}}$ is the angular velocity of the NS.
} 


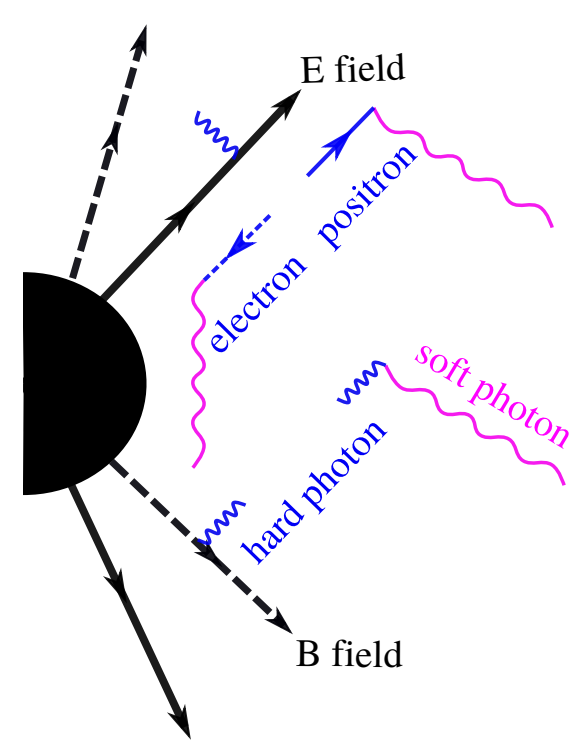

FIG. 1. A cartoon picture summarizing major reactions in our model: electrons and positrons are accelerated in opposite directions by the electric field; soft photons gain energy via IC scatterings with electrons and positrons; $e^{ \pm}$pairs are created as hard photons colliding with background soft photons, or through Schwinger process in electromagnetic fields. Vacuum polarization in charged BHs was originally considered in [21].

Compton (IC) scattering with surrounding soft photons. More electrons and positrons will be generated as a result of pair production of the hard photons interacting with soft photons and electromagnetic fields (see Fig. 1). The above cascade stops only when the charged $\mathrm{BH}$ neutralizes by accreting enough opposite charges. As we will show later $\sim 10 \%$ of the total energy is carries away by $\sim 10^{41}$ gamma-ray photons with energy $>50 \mathrm{GeV}$, which is the low-energy detection threshold of the MAGIC telescope [26].

We use geometric units $G=c=1$ and the Schwarzschild metric is written as

$$
d s^{2}=-\alpha^{2} d t^{2}+\alpha^{-2} d r^{2}+r^{2} d \Omega^{2},
$$

where $\alpha:=\sqrt{1-2 M / r}$ and we assume $M=10 M_{\odot}$ in this paper. Tortoise coordinate $r_{*} \equiv r+2 M \ln \left|\frac{r}{2 M}-1\right|$ is used in our numerical simulations.

\section{GOVERNING EQUATIONS}

The single particle distribution function $f(t, r, p)$ in phase space is defined by counting the particle number $d N$ within the volume $(r, r+d r)$ and $(p, p+d p)$ : $d N=f(t, r, p) 4 \pi \alpha^{-2} d r d p=f(t, r, p) 4 \pi d r_{*} d p$, where $p$ is the contravariant component of momentum in the $r$ direction $p \equiv p^{r}=\sqrt{\left(p_{t}\right)^{2}-\alpha^{2} m^{2}}$, with $-p_{t}$ being the particle energy measured by observers at infinity and $m$ being the particle mass. Boltzmann equation in the covariant form is written as [e.g., 27]

$$
p^{\mu} \frac{\partial f}{\partial x^{\mu}}-\Gamma_{\mu \nu}^{i} p^{\mu} p^{\nu} \frac{\partial f}{\partial p^{i}}+e F^{\mu \nu} p_{\nu} \frac{\partial f}{\partial p^{\mu}}=C[f],
$$

where $\Gamma_{\mu \nu}^{i}$ is the metric connection; $F^{\mu \nu}$ is the Maxwell tensor; $\mu, \nu$ are spacetime indices and $i$ is a spatial index. For the system of spherical symmetry we are considering, the Boltzmann equations for distribution functions of electrons $f_{+}\left(t, r, p_{+}\right)$, positrons $f_{-}\left(t, r, p_{-}\right)$and hard photons $f_{\gamma}\left(t, r, p_{\gamma}\right)$ are written as

$$
\begin{aligned}
\partial_{t} f_{+}\left(p_{+}\right)+v\left(p_{+}\right) \partial_{r_{*}} f_{+}\left(p_{+}\right)+a_{+} \partial_{p} f_{+}\left(p_{+}\right) & =C_{+}, \\
\partial_{t} f_{-}\left(p_{-}\right)+v\left(p_{-}\right) \partial_{r_{*}} f_{-}\left(p_{-}\right)+a_{-} \partial_{p} f_{-}\left(p_{-}\right) & =C_{-}, \\
\partial_{t} f_{\gamma}\left(p_{\gamma}\right)+v\left(p_{\gamma}\right) \partial_{r_{*}} f_{\gamma}\left(p_{\gamma}\right) & =C_{\gamma},
\end{aligned}
$$

where $v(p)=p /\left(-p_{t}\right) ; a_{ \pm}= \pm \alpha^{2} N_{Q} e^{2} / r^{2} m_{e}$ are the acceleration of positrons and electrons in the electric field, with $N_{Q}(r) e$ being the total charge enclosed by radius $r$. Here we have ignored the gravitational force term which is much smaller than the electric force term. The collision terms $C_{ \pm, \gamma}$ are contributed by IC scatterings and pair productions (see Fig. 1),

$$
\begin{aligned}
C_{+}= & -\mathcal{R}_{\mathrm{IC}}\left(p_{+}\right) f_{+}\left(p_{+}\right)+\mathcal{R}_{\mathrm{IC}}\left(p_{+}^{\text {old }}\right) f_{+}\left(p_{+}^{\text {old }}\right) \frac{d p_{+}^{\text {old }}}{d p_{+}} \\
& +\mathcal{R}_{\text {pair }}\left(p_{\gamma}^{\text {old }}\right) f_{\gamma}\left(p_{\gamma}^{\text {old }}\right) \frac{d p_{\gamma}^{\text {old }}}{d p_{+}} \\
C_{-}= & -\mathcal{R}_{\mathrm{IC}}\left(p_{-}\right) f_{-}\left(p_{-}\right)+\mathcal{R}_{\mathrm{IC}}\left(p_{-}^{\text {old }}\right) f_{-}\left(p_{-}^{\text {old }}\right) \frac{d p_{-}^{\text {old }}}{d p_{-}} \\
& +\mathcal{R}_{\text {pair }}\left(p_{\gamma}^{\text {old }}\right) f_{\gamma}\left(p_{\gamma}^{\text {old }}\right) \frac{d p_{\gamma}^{\text {old }}}{d p_{-}} \\
C_{\gamma}= & -\mathcal{R}_{\text {pair }}\left(p_{\gamma}\right) f_{\gamma}\left(p_{\gamma}\right)+\mathcal{R}_{\mathrm{IC}}\left(p_{+}^{\text {old }}\right) f_{+}\left(p_{+}^{\text {old }}\right) \frac{d p_{+}^{\text {old }}}{d p_{\gamma}} \\
& +\mathcal{R}_{\mathrm{IC}}\left(p_{-}^{\text {old }}\right) f_{-}\left(p_{-}^{\text {old }}\right) \frac{d p_{-}^{\text {old }}}{d p_{\gamma}}
\end{aligned}
$$

where $\mathcal{R}_{\mathrm{IC}}$ is the Compton scattering rate of a lepton in the soft photon background, and $\mathcal{R}_{\text {pair }}$ is the total pair production rate of a hard photon colliding with soft photons and through Schwinger process in strong electromagnetic fields $\left(\gamma^{\text {hard }}+\gamma^{\text {soft }} / E / B \rightarrow e^{+}+e^{-}\right)$, i.e., $\mathcal{R}_{\text {pair }}=\mathcal{R}_{\gamma \gamma}+\mathcal{R}_{\gamma E}+\mathcal{R}_{\gamma B}$. On the right hand side of Eq. (5a), $p_{+}^{\text {old }}$ is the momentum of positrons that slow down to $p_{+}$after one IC scattering; $p_{\gamma}^{\text {old }}$ is the momentum of hard photons that annihilate into an $e^{ \pm}$pairs with momentum $p_{+}$, i.e.,

$$
\begin{array}{ll}
p_{+}^{\text {old }}=\frac{p_{+}}{1-\alpha^{-2} E_{\gamma}^{\text {soft }} p_{+} / m_{e}^{2}} & \text { (for Inverse Compton) } \\
p_{\gamma}^{\text {old }}=2 p_{+}=2 p_{-} & \text {(for pair production) },
\end{array}
$$

where $E_{\gamma}^{\text {soft }}:=-p_{t, \gamma}^{\text {soft }}$ is the soft photon energy measured by observers at infinity, and the factor $\alpha^{-2}$ takes account 
of the gravitational redshift in the vicinity of the BH. In Eqs. $(5 \mathrm{~b}, 5 \mathrm{c}), p_{ \pm, \gamma}^{\text {old }}$ are defined in a similar way.

To close the Boltzmann equations (4), we also need the sourced Maxwell's equations, which are written in terms of $N_{Q}$ and $f_{ \pm}(p)$ as follow,

$$
\begin{aligned}
\partial_{t} N_{Q} & =-4 \pi \int_{-\infty}^{\infty} v(p)\left[f_{+}(p)-f_{-}(p)\right] d p, \\
\partial_{r_{*}} N_{Q} & =4 \pi \int_{-\infty}^{\infty}\left[f_{+}(p)-f_{-}(p)\right] d p,
\end{aligned}
$$

where Eq. (7b) is a constraint of charge conservation, instead of an evolution equation. To ensure the charge conservation during evolution, we in fact evolve two extended Maxwell's equations in simulations (see Ref. [28] and references therein for more details).

\section{MODEL SETUP}

We take the moment of BH-NS merger as our starting point $t=0$, when we expect a charged $\mathrm{BH}$ and a remnant NS magnetosphere, as well as some charged particles outside the BH. In this paper, we focus on investigating the $\mathrm{BH}$ discharge process, with simplified assumptions of other relevant processes. Here we do not do a full Boltzmann+Maxwell analysis for the remnant NS magnetosphere, instead we assume the remnant NS magnetopshere is detached from the $\mathrm{BH}$ and expands outward after the merger. ${ }^{2}$

The initial distribution functions $f_{ \pm, \gamma}(r, p)$, electric charge $Q$ carried by the $\mathrm{BH}$ (or equivalently the dimensionless quantity $\Gamma_{Q}$ ) and all the reaction coefficients: $\mathcal{R}_{\mathrm{IC}}, \mathcal{R}_{\gamma \gamma}, \mathcal{R}_{\gamma E}, \mathcal{R}_{\gamma B}$ are specified as follows.

\section{A. remnant magnetopshere and initial conditions}

Motivated by the dipole geometry of magnetic field lines carried by the NS, we assume an initial magnetic

\footnotetext{
2 The fate of the remnant NS magnetosphere is still an open question now. We expect an isolated $\mathrm{BH}$ cannot sustain a magnetosphere according to the "no-hair" theorem. There was some counterargument (e.g., in Ref. [29]) claiming that the "no-hair" theorem does not apply here because they found an isolated $\mathrm{BH}$ can sustain the captured magnetic field for a long time assuming the $\mathrm{BH}$ is able to self-produce a highly conducting plasma and maintain a high magnetization. It is not clear whether the crucial assumption adopted in their simulation, i.e., dissipationless ideal MHD and force-free plasma, is physically realistic as dissipative plasma processes such as pair creation are evitable in this scenario. Slight different assumptions lead to completely different conclusions even for the non-spinning BH case [29, 30].

Even the claim that the $\mathrm{BH}$ can sustain the captured magnetic field for a long time was true, the net $\mathrm{BH}$ charge in such state is about $0.01 a^{3} B_{\mathrm{NS}} r_{\mathrm{NS}}^{2}$ as estimated in Ref. [17], which is much smaller than the amount the $\mathrm{BH}$ acquires in the merger. Here $a$ is the dimensionless $\mathrm{BH}$ spin. Therefore, $\mathrm{BH}$ discharge process is inevitably in either scenario.
}

field of the remnant NS magnetosphere

$$
\left.B(r)\right|_{t=0}=B_{\mathrm{NS}}\left(r_{\mathrm{NS}} / r\right)^{3} \quad\left(\text { for } r>r_{c}=6 M\right) .
$$

The soft photon energy and number density are estimated assuming they mainly come from cyclotron radiation by the non-relativistic leptons (with GoldreichJulian density [31]) in the magnetic field, i.e.,

$$
\begin{aligned}
E_{\gamma}^{\mathrm{soft}} & =\hbar \omega_{B}=\hbar \frac{e B}{m_{e}}, \\
n_{\gamma}^{\mathrm{soft}}(r) & \approx \int_{r_{c}}^{\infty} n_{\mathrm{GJ}}\left(r^{\prime}\right) f_{\mathrm{cyc}}\left(r^{\prime}\right) \frac{r^{\prime 2}}{\left|\overrightarrow{r^{\prime}}-\vec{r}\right|^{2}} d r^{\prime},
\end{aligned}
$$

where $n_{\mathrm{GJ}}=0.07(B / \mathrm{G}) \mathrm{cm}^{-3}$ is the Goldreich-Julian number density (for a NS magnetosphere with rotation period of $1 \mathrm{sec}$ ), $f_{\text {cyc }} \approx \frac{2}{3} \frac{e^{2}}{\hbar c} \omega_{B}$ is the number of soft photons emitted per unit time from a non-relativistic lepton moving in the magnetic field.

The energy of soft photons varies from maximum value $\sim 10 \mathrm{eV}$ to zero depending on the magnitude of underlying magnetic field. For our purpose here, only the soft photons at the high-energy end affect both emission and absorption of hard photons. Therefore, we assume all soft photons are of the same energy $E_{\gamma}^{\text {soft }} \in[1,10] \mathrm{eV}$.

For a given $\Gamma_{Q}$, we set $\left.f_{+}\right|_{t=0}=\left.f_{\gamma}\right|_{t=0}=0$ and

$$
\left.f_{-}\right|_{t=0} d p=\frac{n(r)}{\sqrt{2 \pi} \sigma_{p}} \exp \left(-\frac{\left(p / \Gamma_{Q} m_{e}\right)^{2}}{2 \sigma_{p}^{2}}\right) d\left(\frac{p}{\Gamma_{Q} m_{e}}\right),
$$

with $\sigma_{p}=0.1$ and $n(r) / r^{2}=c_{n} /\left(\exp \left(\left(r_{*}-6 M\right) / 2 M\right)+\right.$ 1 ), where $c_{n}$ is some normalization constant ensuring electric neutrality at infinity. As we will show later, the discharge process and final products mainly depend on the initial amount of total electric energy, while the initial distribution function $f_{-}$does not affect the evolution much as long as the initial kinetic energy of electrons is a minor component.

\section{B. reaction coefficients}

As a result of Eq. (9), we can parameterize the rate of a lepton scattered off soft photons as

$$
\mathcal{R}_{\mathrm{IC}}=n_{\gamma}^{\mathrm{soft}} \sigma_{\mathrm{T}}=\left(10 N_{\gamma}^{\mathrm{soft}}\right) \times \alpha^{2} r_{c}^{5} /\left(r_{c}^{5}+r^{5}\right) M^{-1},
$$

where $\sigma_{\mathrm{T}}$ is the Thomson cross section. $N_{\gamma}^{\text {soft }}$ is a free parameter of $O(1)$ and the factor $\alpha^{2}$ is included to mimic the lower photon number density in the vicinity of the $\mathrm{BH}$ due to $\mathrm{BH}$ capturing.

For the soft photon background above, the coefficient $\mathcal{R}_{\gamma \gamma}$ is written as $\mathcal{R}_{\gamma \gamma}=\xi_{\gamma \gamma}\left(p, E_{\gamma}^{\text {soft }}\right)\left(\sigma_{\gamma \gamma} / \sigma_{\mathrm{T}}\right) \mathcal{R}_{\mathrm{IC}}$, where $\sigma_{\gamma \gamma} \approx 0.35 \sigma_{\mathrm{T}}$ is the cross section of pair creation $\gamma+\gamma \rightarrow e^{+}+e^{-}[32]$ and factor $\xi_{\gamma \gamma}$ takes account of the energy threshold below which this process is prohibited. The pair creation coefficients $\mathcal{R}_{\gamma E}$ and $\mathcal{R}_{\gamma B}$ are known as [e.g., 33]

$$
\mathcal{R}_{\gamma X}=\frac{0.23}{a_{0}} X^{\prime} \exp \left(-\frac{4}{3 x X^{\prime}}\right),
$$


where $X=\{E, B\}, a_{0}$ is the Bohr radius, $x=p_{\gamma} / 2 m_{e}$, $X^{\prime}=X / X_{\text {cr }}$ and $X_{\text {cr }} \equiv m^{2} c^{3} / \hbar e=4.4 \times 10^{13} \mathrm{G}$. In our model, we obtain electric field $E(t, r)$ self consistently and assume the remnant magnetosphere expands outward in the light speed which determines the evolution of magnetic field $B(t, r)$ as ${ }^{3}$

For notation simplicity, we define a two-element array $\mathcal{R}_{\gamma \#}:=\left\{\left.\mathcal{R}_{\gamma E}\right|_{E=\left(N_{Q} e / r^{2}\right)\left(\Gamma_{Q}, \# / \Gamma_{Q}\right)},\left.\mathcal{R}_{\gamma B}\right|_{B_{\mathrm{NS}}=10 \# \mathrm{G}}\right\}$ for cases with $B_{\mathrm{NS}}=10^{\#} \mathrm{G}$, e.g., $\mathcal{R}_{\gamma 12}$ is a short-hand notation for $\left\{\left.\mathcal{R}_{\gamma E}\right|_{E=\left(N_{Q} e / r^{2}\right)\left(\Gamma_{Q, 12} / \Gamma_{Q}\right)},\left.\mathcal{R}_{\gamma B}\right|_{B_{\mathrm{NS}}=10^{12} \mathrm{G}}\right\}$ for cases with $B_{\mathrm{NS}}=10^{12} \mathrm{G}$.

\section{C. implicit assumptions}

A few extra assumptions have been made in the model setup and we summarize them as follows.

Compton scatterings of leptons with hard photons are not included considering the lower number density of hard photons compared with that of soft photons and the smaller cross section which is suppressed by a factor $\sim p_{ \pm} p_{\gamma} / m_{e}^{2}$ in high-energy limit.

We do not track the evolution of soft photons in the Boltzmann equations, instead we approximate them as a background field with number density $n_{\gamma}^{\text {soft }}(r)$ solely determined by cyclotron radiation from the remnant magnetosphere, since the optical depth of soft photons due to collisions with high-energy pairs and hard photons $\sim n_{ \pm, \gamma} \sigma_{\mathrm{T}} M$ is much smaller than unity for the problem we are considering.

The detailed evolution of the remnant magnetosphere is another controversial problem even for non-rotating remnant BHs [e.g., 29, 30]. In spite of these certainties, one certain thing is that the remnant magnetopshere is magnetically dominant, $B=B_{r} \gg E_{\perp}$, where $E_{\perp}$ is the perpendicular E-field component ensuring another Maxwell's equation $\partial_{t} \vec{B}_{r}=-\nabla \times \vec{E}_{\perp}$. In this paper, we have ignored the small component $E_{\perp}$, and only keep $E_{r}$ and $B_{r}$.

${ }^{3}$ While the exact expansion speed is unknown, the propagation velocities of characteristic electromagnetic waves in the remnant magnetosphere are close to light speed. Considering different directions of these waves move in, we expect the remnant magnetosphere expand in a sub-light speed, whose exact value barely affects the discharge process.

$$
B(t, r)=B_{\mathrm{NSS}} \frac{r_{\mathrm{NS}}^{3}}{(r-t)^{3}}\left(\frac{r-t}{r}\right)^{4 / 3}\left(\text { for } r-t>r_{c}\right)
$$

\section{BH DISCHARGE AND VERY-HIGH-ENERGY GAMMA RAYS}

\section{A. numerical setup}

To evolve the governing equations $(4,7 \mathrm{a}, 7 \mathrm{~b})$, we use the 4-th order Runge-Kutta method on a $512 \times 128$ grid (default resolution) covering the phase space of $\left[r_{*, \min }, r_{*, \max }\right] \times\left(\left[-p_{\max },-p_{\min }\right] \bigoplus\left[p_{\min }, p_{\max }\right]\right)$, where we take $r_{*, \min }=-8.6 M, r_{*, \max }=56.5 M$ (corresponding to $\left.r_{\min }=2.01 M, r_{\max }=50 M\right)$, and $p_{\max }=$ $\Gamma_{Q} m_{e}, p_{\min } \lesssim 0.01 m_{e}^{2} / E_{\gamma}^{\text {soft }}$.

We use the ingoing boundary condition at "horizon" $r=r_{*, \text { min }}$ and the outgoing boundary condition at "infinity" $r=r_{* \text {,max }}$, i.e., no particle enters the computation domain from two boundaries. For the boundaries in the $p$ direction, we naturally choose $f_{ \pm, \gamma}\left(p= \pm p_{\max }\right)=0$, i.e., no particle is accelerated to the maximum energy $\Gamma_{Q} m_{e}$.

The convergence test and conservation test for our code have been done for a fiducial model and are shown in the Appendix.

\section{B. simulation results}

We choose a fiducial model with $\Gamma_{Q}=3 \times 10^{8}, E_{\gamma}^{\text {soft }}=$ $10 \mathrm{eV}, N_{\gamma}^{\text {soft }}=1,\left\{\mathcal{R}_{\gamma E}, \mathcal{R}_{\gamma B}\right\}=\mathcal{R}_{\gamma 12}$, and show the detailed discharge process in Fig. 2. In the region where electric field is not screened, electrons and positrons are accelerated and then scattered by soft photons in a distance $1 / \mathcal{R}_{\mathrm{IC}} \sim 0.1 M$. Hard photons produced by IC scatterings are depleted by the electromagnetic fields immediately via Schwinger process $\gamma+E / B \rightarrow e^{+}+e^{-}$. As a result, number of $e^{ \pm}$pairs increases exponentially while number of hard photons stays at a relatively low level due to $\mathcal{R}_{\text {pair }} \gg \mathcal{R}_{\text {IC }}$ (see 1st row of Fig. 2). After a few folds of exponential growth, local number density of pairs exceeds the initial charge density, i.e., $n_{+} \approx n_{-} \gg\left|n_{+}-n_{-}\right|$, and electric field is screened by electrons and positrons accelerated in opposite directions (see 2nd row of Fig. 2). As the exponential growth continues, electric field is screened over larger and larger area, leaving a window where EM fields vanish and hard photons survive the Schwinger process. In the EM-free window, leptons and hard photons are driven towards lower energy by colliding with soft photons (see 3rd row of Fig. 2).

We measure the total number of very-high-energy (VHE) gamma rays escaping to infinity

$$
N_{\gamma}^{\mathrm{VHE}}=\left.4 \pi \int_{p>50 \mathrm{GeV}} f_{\gamma}(t, r, p)\right|_{r \rightarrow \infty} d t d p,
$$

and the energy spectrum $d N_{\gamma}^{\mathrm{VHE}} / d p$. We find the energy spectrum is roughly a power law $d N_{\gamma}^{\mathrm{VHE}} / d p \propto p^{-2.3}$ plus a bump at $p \approx m_{e}^{2} / E_{\gamma}^{\text {soft }}$, which is the energy threshold of hard photons colliding with soft photons and producing $e^{ \pm}$pairs (see Fig. 3). 

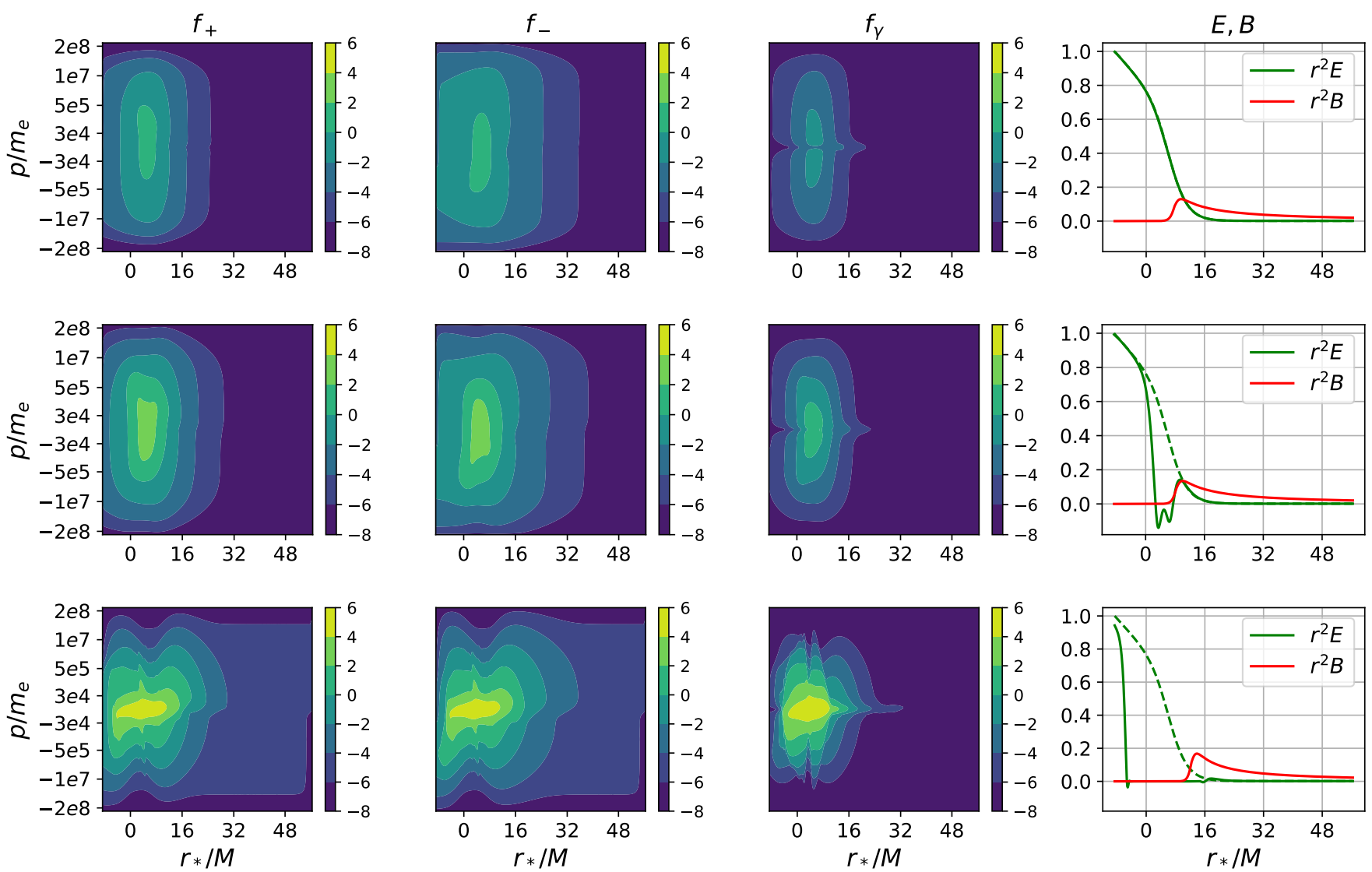

FIG. 2. BH discharge process of a fiducial model with parameters $\Gamma_{Q}=3 \times 10^{8}, E_{\gamma}^{\text {soft }}=10 \mathrm{eV}, N_{\gamma}^{\text {soft }}=1$ and $\left\{\mathcal{R}_{\gamma E}, \mathcal{R}_{\gamma B}\right\}=$ $\mathcal{R}_{\gamma 12}$. Top/Middle/Bottom rows are snapshots at $t=0.2 M / 0.6 M / 3.7 M$, respectively. Left three columns are the distribution functions $f_{ \pm, \gamma}$ (more accurately $\log _{10}\left[f_{ \pm, \gamma} \cdot\left(M \Gamma_{Q} m_{e}\right)\right]$ ). In the right panel, we plot EM fields $r^{2} E$ and $r^{2} B$ in unit of $M^{2} \times 10^{12} \mathrm{G}$, where the dashed lines are the initial value of $r^{2} E$.

In Fig. 4, we detail the dependence of $N_{\gamma}^{\mathrm{VHE}}$ on the four model parameters, which is approximately described by $N_{\gamma}^{\mathrm{VHE}}=N_{0}\left(E_{\gamma}^{\mathrm{soft}}, N_{\gamma}^{\mathrm{soft}}, B_{\mathrm{NS}}\right) \times\left(\Gamma_{Q}\right)^{2}$, where $\left.N_{0}\right|_{p>50 \mathrm{GeV}} \approx 10^{12}$ (and $\left.\left.N_{0}\right|_{p>20 \mathrm{GeV}} \approx 3 \times 10^{12}\right)$. The initial $\mathrm{BH}$ charge sets the total energy budget $\left(\propto \Gamma_{Q}^{2}\right)$ for particle creation, which explains the dependence of $N_{\gamma}^{\mathrm{VHE}}$ on $\Gamma_{Q}$. The soft photon energy $E_{\gamma}^{\mathrm{soft}}$ sets an threshold $m_{e}^{2} / E_{\gamma}^{\text {soft }}$ below which hard photons freely stream in the soft photon background without pair creation. Therefore the smaller $E_{\gamma}^{\text {soft }}$, the higher threshold and the more hard photons escaping to infinity. The number density of soft photons $N_{\gamma}^{\text {soft }}$ determines the mean free path of leptons $\left(1 / \mathcal{R}_{\text {IC }}\right)$ and that of hard photons $\left(1 / \mathcal{R}_{\gamma \gamma}\right)$, where the former determines the amount of energy a lepton can gain from the electric field and the latter determines the annihilation possibility of a hard photon. Therefore the larger $N_{\gamma}^{\text {soft }}$, the less energy carried away to infinity by VHE photons. The magnetic field strength $B_{\mathrm{NS}}$ (or equivalently the coefficient $\mathcal{R}_{\gamma \#}$ ) mainly affects the width of window in which EM fields vanish and hard photons survive the Schwinger process. We expect this to be a marginal effect which is confirmed by our simulations.
Now let us examine the detection prospect for the $\mathrm{BH}$ discharge process after a typical type III BH-NS merger. For very short observation time, the sensitivity of a Cherenkov telescope is limited by number $N_{\text {obs }}$ of high-energy particles arriving at the telescope. Using the commonly used detection criteria $N_{\text {obs }}>10$ [26], we obtain the horizon distance

$$
d=\left(\frac{N_{\gamma}^{\mathrm{VHE}}}{1.2 \times 10^{41}}\right)^{1 / 2}\left(\frac{S}{1 \mathrm{~km}^{2}}\right)^{1 / 2} \mathrm{Mpc}
$$

within which $\mathrm{BH}$ discharge events are visible to the Cherenkov telescope, where the effective area $S \sim 5 \mathrm{~km}^{2}$ for the telescope proposed in [34].

Our simulation is unable to trace the lowest energy photons due to limited resolution. Assuming hard photons of energy $100 \mathrm{MeV}$ carry away total energy $5 \times 10^{41}$ ergs, the horizon distance for Fermi LAT [35] (with effective area $\sim 0.35 \mathrm{~m}^{2}$ at this energy) is about $0.1 \mathrm{Mpc}$. 


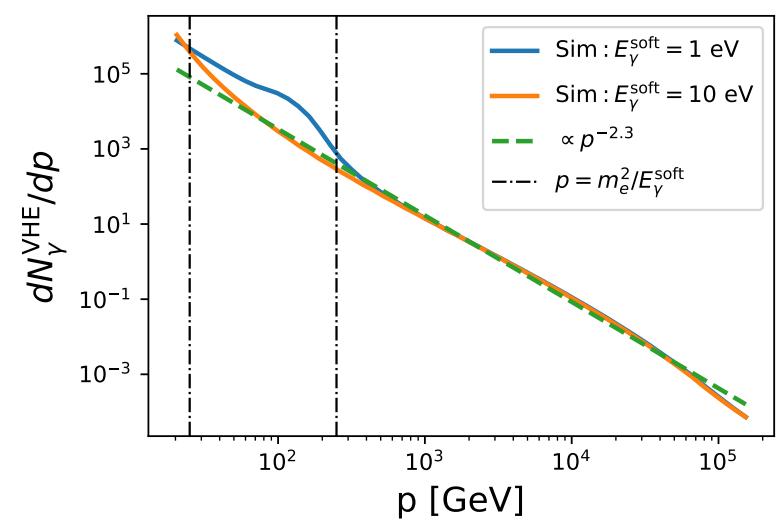

FIG. 3. The energy spectrum of VHE gamma-rays observed from far-away for a model with parameters $\Gamma_{Q}=3 \times 10^{8}$, $E_{\gamma}^{\text {soft }}=\{1,10\} \mathrm{eV}, N_{\gamma}^{\text {soft }}=1$ and $\left\{\mathcal{R}_{\gamma E}, \mathcal{R}_{\gamma B}\right\}=\mathcal{R}_{\gamma 12}$, where $d N_{\gamma}^{\mathrm{VHE}} / d p$ is given in arbitary units.

\section{SUMMARY AND DISCUSSION}

\section{A. Summary}

In this paper, we perform a first-principle study of EM counterparts of type III BH/NS mergers, where the $\mathrm{BH}$ is large enough to swallow the NS as a whole without star disruption. In the late inspiral stage, the $\mathrm{BH}$ is charged as moving in the NS magnetosphere. The remnant $\mathrm{BH}$ neutralizes rapidly via a discharge process, through which the electric energy is converted into the kinetic energy of $e^{+} / e^{-}$pairs and hard photons. For a typical BH/NS merger with $M=10 M_{\odot}, B_{\mathrm{NS}}=10^{12} \mathrm{G}$ and $r_{\mathrm{NS}}=10$ $\mathrm{km}$, we find that $\sim 10 \%$ of the total electric energy is carried away to infinity in a time interval $\sim 1 \mathrm{~ms}$ by $\mathrm{VHE}$ gamma rays, whose spectrum is approximately a power law $d N_{\gamma}^{\mathrm{VHE}} / d p \propto p^{-2.3}$ plus a bump at $p \approx m_{e}^{2} / E_{\gamma}^{\mathrm{soft}}$ (see Fig. 3).

In reality, soft photons from cyclotron radiation in the non-uniform magnetic field are not mono-energetic. As shown in Figs. 3 and 4, different soft photon energy indeed mildly changes the total number and the energy spectrum of VHE photons. We expect a mild increase in the total number $N_{\gamma}^{\mathrm{VHE}}$ and flatter bump in the spectrum $d N_{\gamma}^{\mathrm{VHE}} / d p$ if the low-energy tail of soft photons are taken account of.

We may similarly estimate the EM signal from a $\mathrm{BH} /$ magnetar merger. For a magnetar with magnetic field $10^{15} \mathrm{G}$ [36, 37], the final $\mathrm{BH}$ would be charged to $10^{3} Q_{, 12}$ with electric energy $\sim 10^{48}$ ergs. Soft photon energy $\left(\hbar \omega_{B}\right)$ would be $10^{3}$ larger, and typical hard photon energy $m_{e}^{2} / E_{\gamma}^{\text {soft }} \sim 25 \mathrm{MeV}$. Therefore we expect a dim sGRB after a $\mathrm{BH} /$ magnetar merger which is visible to Fermi LAT within $\sim 100 \mathrm{Mpc}$. The discharging process discussed here also sheds light on the EM signature of charged BH mergers [19, 38] and/or charged BHs formed through gravitational collapse of magnetized NSs [30].

\section{B. Discussion}

It is beneficial to compare the $\mathrm{BH}$ discharge process with that of a NS. As shown in [e.g., Ref. 20], a spinning NS carrying a magnetosphere is also charged with electric charge $Q_{\mathrm{NS}} \sim \Omega_{\mathrm{NS}} B_{\mathrm{NS}} r_{\mathrm{NS}}^{3}$, where $\Omega_{\mathrm{NS}}$ is the NS angular velocity. The NS magnetosphere is approximately being force-free (the component of the electric field parallel to the magnetic field $E_{\|}$vanishes), except in some special regions, e.g., the polar caps. Inside the polar cap, a cascade of pair creation and synchrotron radiation is driven by the strong unscreened electric field $E_{\|}[39,40]$. As a result, electrons and positrons are accelerated by the parallel electric field $E_{\|}$in opposite directions. This process is very similar to the $\mathrm{BH}$ discharge studied in this paper. However, the cascade in the NS polar caps does not continually decrease the NS charge $Q_{\mathrm{NS}}$ due to the existence of a recovery force $B_{\mathrm{NS}}$ which is unaffected by the cascade and drive a electric current circuit along open magnetic field lines keeping both the electric field and the NS charge in the equilibrium state. To summarize, it is the NS magnetic field $B_{\mathrm{NS}}$ that keeps the NS from being discharged.

An essential difference between a (isolated) $\mathrm{BH}$ and a NS is that the $\mathrm{BH}$ cannot sustain the magnetic field captured from e.g., a BH-NS merger. Unlike NSs which are almost perfect conductors capable of trapping magnetic fields, BHs are dissipative according to the membrane paradigm [41]. Therefore we do not expect longlived magnetic fields around black holes, if there is no external driving source. If a cascade of pair creation and high-energy photon production is ignited, the initial $\mathrm{BH}$ charge $Q_{\mathrm{BH}}$ will inevitably deplete because there is no such recovery force that plays the role of replenishing current circuit as in the spinning NS case. Both electric and magnetic fields decrease monotonically as the $\mathrm{BH}$ charge decreases.

Of course, the $\mathrm{BH}$ discharge picture will be completely different if we are considering a spinning $\mathrm{BH}$ immersed in a magnetic field $B_{\text {ext }}$ sourced by some external electric current, e.g., sustained by a conducting accretion flow. In this case, the $\mathrm{BH}$ will not be discharged by the cascade as the magnetic field $B_{\text {ext }}$ now serves as the recovery force maintaining both the $\mathrm{BH}$ and the magnetosphere in the charged state.

Throughout this work, we have assumed spherical symmetry for the system of charged $\mathrm{BH}$ and surrounding particles, and have solved the Boltzmann equations of $1+1$ form. The key elements of the $\mathrm{BH}$ discharge process are the initial BH charge (energy budget) and the remnant NS magnetosphere (soft photon source). For a Kerr-Newman BH, there will be both electric and magnetic fields measured by, e.g., Carter observers [42]. Both fields $\left(E \sim Q / r^{2}\right.$ and $B \sim 2 a M Q / r^{3}$ with $a$ being the dimensionless $\mathrm{BH}$ spin [43]) are proportional to the $\mathrm{BH}$ 

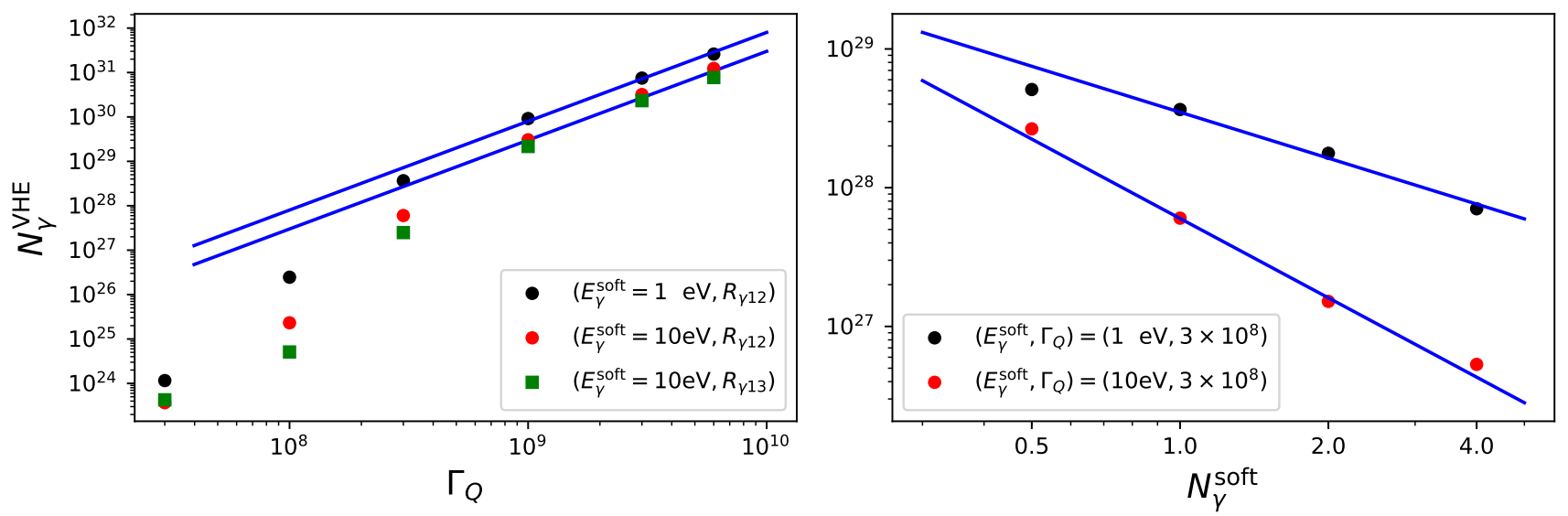

FIG. 4. Dependence of VHE photon number $N_{\gamma}^{\mathrm{VHE}}$ on various model parameters, which take fiducial values if not explicitly specified. In the left panel, we show the relation of $N_{\gamma}^{\mathrm{VHE}}$ with initial BH charge $\Gamma_{Q}$, which is fitted by $N_{\gamma}^{\mathrm{VHE}}=N_{0} \times\left(\Gamma_{Q}\right)^{2}$, with $N_{0} \approx 10^{12}$. In the right panel, we show dependence of $F_{\gamma}^{\mathrm{VHE}}$ (fraction of initial electric energy carried away to infinity by VHE photons) on soft photon number density $N_{\gamma}^{\text {soft }}$, which is roughly $F_{\gamma}^{\mathrm{VHE}} \propto\left(N_{\gamma}^{\mathrm{soft}}\right)^{-\xi}$, with power index $\xi$ being $O(1)$.

charge and will be screened by the leptons in a similar way as in the non-spinning $\mathrm{BH}$ case. In a naive picture, the Kerr-Newman BH is approximately a charged spinning object of size $\sim M$ and carrying both net charge and a current loop, which source the E-field and the B-field, respectively. With the ignition of pair production, particles of opposite charge are attracted towards the central $\mathrm{BH}$ until the total charge is neutralized and therefore the E-field is screened. While the charged particles move inward, they are forced to rotate with the B-field lines and generate an opposite current loop until the initial current loop is counteracted and therefore the B-field is screened. A non-zero BH spin does not introduce any recovery force as in the NS case, therefore we expect the discharge process also to be true for spinning charged BHs. To understand the details of 3D discharge process for spinning BHs where electrodynamics of higher dimensions kick in, our low-dimension analysis does not work any more and it is preferable to apply the particle-in-cell simulations [44-46].

\section{ACKNOWLEDGMENTS}

We would like to thank the referees for their comments which enable a substantial improvement of this paper.
We thank Junwu Huang and Luis Lehner for very helpful discussions. This research was also supported by the Natural Sciences and Engineering Research Council of Canada and in part by Perimeter Institute for Theoretical Physics. Research at Perimeter Institute is supported in part by the Government of Canada through the Department of Innovation, Science and Economic Development Canada and by the Province of Ontario through the Ministry of Economic Development, Job Creation and Trade.

\section{Appendix: Sanity Check}

As a sanity check of our simulations, we run a convergence test and a conservation test for the fiducial model (see Fig. 2 for the model parameters). For the convergence test, we evolve the governing equations with three different resolutions and find the simulations are well converged for the default medium resolution we used throughout this paper (see Fig. 5 for a glimpse). For the conservation test, we keep track of the total energy $E_{\text {tot }}$ of the whole system, including energy of the electric field, energy of all particles residing in the computation domain and energy of particles captured by the central BH or escaping to infinity. We find the energy conservation is satisfied with percent-level precision.
[1] LIGO/Virgo Scientific Collaboration, Phys. Rev. Lett. 119, 161101 (2017), arXiv:1710.05832.

[2] LIGO/Virgo Scientific Collaboration, Astrophys. J. 848, L12 (2017).

[3] H.-T. Janka, T. Eberl, M. Ruffert, and C. L. Fryer, Astrophys. J. 527, L39 (1999), arXiv:9908290 [astro-ph].
[4] H. Miao, H. Yang, and D. Martynov, Phys. Rev. D98, 044044 (2018), arXiv:1712.07345 [gr-qc].

[5] D. Martynov, H. Miao, H. Yang, F. H. Vivanco, E. Thrane, R. Smith, P. Lasky, W. E. East, R. Adhikari, A. Bauswein, et al., arXiv preprint arXiv:1901.03885 (2019). 

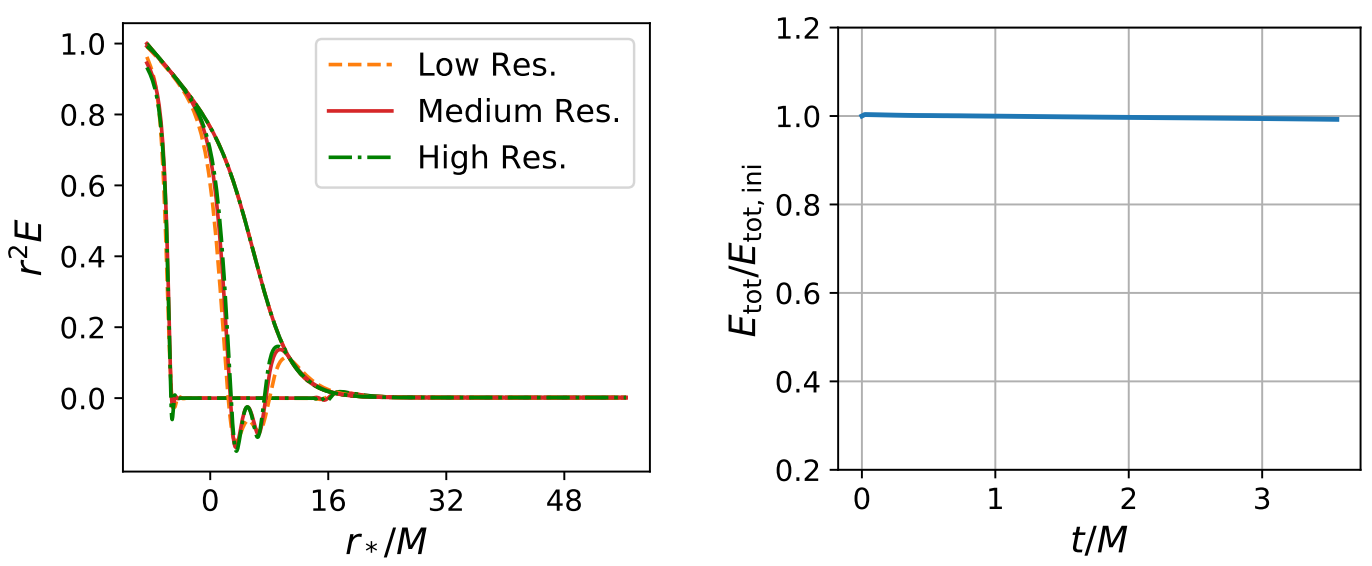

FIG. 5. In the left panel, we show the discharge process at time $t=0 / 0.6 / 3.7 M$ for three different resolutions. In the right panel, we show the precision of energy conservation for the simulation with the default medium resolution, where the total energy $E_{\text {tot }}$ is the total energy of the whole system, including energy of the electric field, and energy of high-energy pairs and photons (residing in the computation domain and escaping to infinity).

[6] H. Yang, W. E. East, and L. Lehner, Astrophys. J. 856, 110 (2018), [Erratum: Astrophys. J.870,no.2,139(2019)], arXiv:1710.05891 [gr-qc].

[7] T. Hinderer et al., (2018), arXiv:1808.03836 [astroph.HE].

[8] H.-Y. Chen and K. Chatziioannou, (2019), arXiv:1903.11197 [astro-ph.HE].

[9] M. Shibata, K. Kyutoku, T. Yamamoto, and K. Taniguchi, Phys. Rev. D 79, 044030 (2009).

[10] Z. B. Etienne, Y. T. Liu, S. L. Shapiro, and T. W. Baumgarte, Phys. Rev. D 79, 044024 (2009).

[11] K. Kyutoku, H. Okawa, M. Shibata, and K. Taniguchi, Phys. Rev. D 84, 064018 (2011).

[12] F. Foucart, M. D. Duez, L. E. Kidder, M. A. Scheel, B. Szilagyi, and S. A. Teukolsky, Phys. Rev. D 85, 044015 (2012).

[13] Z. Mark, H. Yang, A. Zimmerman, and Y. Chen, Phys. Rev. D91, 044025 (2015), arXiv:1409.5800 [gr-qc].

[14] O. J. C. Dias, M. Godazgar, and J. E. Santos, Phys. Rev. Lett. 114, 151101 (2015), arXiv:1501.04625 [gr-qc].

[15] S. T. McWilliams and J. Levin, Astrophys. J. 742, 90 (2011), arXiv:1101.1969.

[16] D. Lai, Astrophys. J. 757, L3 (2012), arXiv:1206.3723 [astro-ph.HE].

[17] J. Levin, D. J. D'Orazio, and S. Garcia-Saenz, Phys. Rev. D 98, 123002 (2018), arXiv:1808.07887.

[18] Z. G. Dai, Astrophys. J. Lett. 873, L13 (2019), arXiv:1902.07939.

[19] B. Zhang, The Astrophysical Journal Letters 827, L31 (2016).

[20] B. Zhang, The Astrophysical Journal Letters 873, L9 (2019), arXiv:1901.11177 [astro-ph.HE].

[21] G. W. Gibbons, Commun. Math. Phys. 44, 245 (1975).

[22] B. Tetarenko, G. Sivakoff, C. Heinke, and J. Gladstone, The Astrophysical Journal Supplement Series 222, 15 (2016).

[23] J. Strader, L. Chomiuk, T. Maccarone, J. Miller-Jones, and A. Seth, Nature 490, 71 (2012), arXiv:1210.0901 [astro-ph.HE].
[24] L. Chomiuk, J. Strader, T. J. Maccarone, J. C. MillerJones, C. Heinke, E. Noyola, A. C. Seth, and S. Ransom, The Astrophysical Journal 777, 69 (2013).

[25] J. C. A. Miller-Jones et al., Mon. Not. Roy. Astron. Soc. 453, 3918 (2015), arXiv:1509.02579 [astro-ph.HE].

[26] J. Aleksic, S. Ansoldi, L. Antonelli, P. Antoranz, A. Babic, et al., Astroparticle Physics 72, 76 (2016).

[27] A. Mezzacappa and R. A. Matzner, Astrophys. J. 343, 853 (1989).

[28] C. Palenzuela, L. Lehner, and S. Yoshida, Phys. Rev. D 81, 084007 (2010).

[29] M. Lyutikov and J. C. McKinney, Phys. Rev. D 84, 084019 (2011).

[30] A. Nathanail, E. R. Most, and L. Rezzolla, Mon. Not. Roy. Astron. Soc. 469, L31 (2017), arXiv:1703.03223 [astro-ph.HE].

[31] P. Goldreich and W. H. Julian, Astrophys. J. 157, 869 (1969).

[32] R. J. Gould and G. P. Schréder, Phys. Rev. 155, 1404 (1967).

[33] J. K. Daugherty and A. K. Harding, Astrophys. J. 273, 761 (1983).

[34] The CTA Consortium, arXiv preprint arXiv:1904.12196 (2019), arXiv:1904.12196 [astro-ph.HE].

[35] W. B. Atwood, A. A. Abdo, M. Ackermann, W. Althouse, et al., Astrophys. J. 697, 1071 (2009), arXiv:0902.1089 [astro-ph.IM].

[36] P. M. Woods, V. M. Kaspi, C. Thompson, F. P. Gavriil, H. L. Marshall, D. Chakrabarty, K. Flanagan, J. Heyl, and L. Hernquist, Astrophys. J. 605, 378 (2004), arXiv:astro-ph/0310575 [astro-ph].

[37] S. Mereghetti, J. Pons, and A. Melatos, Space Sci. Rev. 191, 315 (2015), arXiv:1503.06313 [astro-ph.HE].

[38] T. Liu, G. E. Romero, M.-L. Liu, and A. Li, The Astrophysical Journal 826, 82 (2016).

[39] J. K. Daugherty and A. K. Harding, Astrophys. J. 252, 337 (1982).

[40] B. Zhang and A. K. Harding, Astrophys. J. 532, 1150 (2000), astro-ph/9911028. 
[41] K. S. Thorne, R. H. Price, and D. A. MacDonald, Black Holes: The Membrane Paradigm (1986).

[42] B. Carter, Communications in Mathematical Physics 10, 280 (1968).

[43] R. L. Znajek, Mon. Not. Roy. Astron. Soc. 179, 457 (1977).

[44] A. Levinson and B. Cerutti, Astronomy and Astrophysics 616, A184 (2018), arXiv:1803.04427 [astro-ph.HE].
[45] K. Parfrey, A. Philippov, and B. Cerutti, Phys. Rev. Lett. 122, 035101 (2019), arXiv:1810.03613 [astroph.HE].

[46] A. Y. Chen, Y. Yuan, and H. Yang, Astrophys. J. 863, L31 (2018), arXiv:1805.11039 [astro-ph.HE]. 